\title{
European Stroke Organisation (ESO) guidelines for the management of temperature in patients with acute ischemic stroke
}

\author{
George Ntaios ${ }^{*}$, Tomasz Dziedzic², Patrik Michel ${ }^{3}$, Vasileios Papavasileiou ${ }^{1}$ Jesper Petersson ${ }^{4}$, \\ Dimitre Staykov ${ }^{5,6}$, Brenda Thomas ${ }^{7}$, and Thorsten Steiner ${ }^{8,9}$ for the \\ European Stroke Organisation
}

\begin{abstract}
Background Hyperthermia is a frequent complication in patients with acute ischemic stroke. On the other hand, therapeutically induced hypothermia has shown promising potential in animal models of focal cerebral ischemia. This Guideline Document presents the European Stroke Organisation guidelines for the management of temperature in patients with acute ischemic stroke.

Methods A multidisciplinary group identified related questions and developed its recommendations based on evidence from randomized controlled trials elaborating the Grading of Recommendations Assessment, Development, and Evaluation approach. This Guideline Document was reviewed within the European Stroke Organisation and externally and was approved by the European Stroke Organisation Guidelines Committee and the European Stroke Organisation Executive Committee.

Results We found low-quality evidence, and therefore, we cannot make any recommendation for treating hyperthermia as a means to improve functional outcome and/or survival in patients with acute ischemic stroke and hyperthermia; moderate evidence to suggest against routine prevention of hyperthermia with antipyretics as a means to improve functional outcome and/or survival in patients with acute ischemic stroke and normothermia; very low-quality evidence to suggest against routine induction of hypothermia as a means to improve functional outcome and/or survival in patients with acute ischemic stroke.

Conclusions The currently available data about the management of temperature in patients with acute ischemic stroke are limited, and the strengths of the recommendations are therefore weak. We call for new randomized controlled trials as well as recruitment of eligible patients to ongoing randomized
\end{abstract}

Correspondence: George Ntaios*, Department of Medicine, University of Thessaly, Biopolis 41110, Larissa, Greece.

E-mail: gntaios@med.uth.gr

${ }^{1}$ Department of Medicine, University of Thessaly, Larissa, Greece

${ }^{2}$ Department of Neurology, Jagiellonian University, Krakow, Poland

${ }^{3}$ Stroke center, Neurology Service, CHUV, University of Lausanne, Lausanne, Switzerland

${ }^{4}$ Department of Neurology, Skåne University Hospital, Malmö, Sweden

${ }^{5}$ Department of Neurology, University of Erlangen-Nuremberg, Erlangen, Germany

${ }^{6}$ Department of Neurology, Hospital of the Brothers of St. John, Eisenstadt, Austria

${ }^{7}$ Centre for Clinical Brain Sciences, University of Edinburgh, Edinburgh, UK

${ }^{8}$ Department of Neurology, Klinikum Frankfurt Höchst, Frankfurt, Germany

${ }^{9}$ Department of Neurology, Heidelberg University, Heidelberg, Germany

Received: 4 June 2015; Accepted: 1 June 2015

Conflict of interest: Please refer to Appendix S5 for the list of conflicts of interest.

DOI: $10.1111 /$ ijs. 12579 controlled trials to allow for better-informed recommendations in the future.

Key words: acute ischemic stroke, hyperthermia, hypothermia, normothermia, temperature management

\section{Introduction}

Recently, the European Stroke Organisation (ESO) updated its policy on preparation and publication of clinical guidelines. There have been two major developments: First, it was decided that the ESO would implement the GRADE system as a guide for the preparation of the guidelines because of its advantages over other systems $(1,2)$. Second, it was decided that guidelines would not be prepared and published as a single document but rather in several documents each one focusing on a specific topic of interest (called a 'module'); this approach allows us to address each module in greater detail and depth, and also provides more flexibility to the process which in turn contributes to the aim of delivering guidelines in a timely manner.

Hyperthermia is a frequent complication in up to $50 \%$ of patients with acute ischemic stroke $(3,4)$, and it was shown to be associated with a poor outcome $(5,6)$. On the other end, therapeutically induced hypothermia has shown promising potential in patients with hypoxic encephalopathy and in animal models of focal cerebral ischemia. This document presents the ESO guidelines on temperature management in patients with acute ischemic stroke. The aim of this Guideline document is to assist physicians treating patients with acute ischemic stroke in their clinical decisions with regard to the management of temperature.

\section{Methods}

The ESO Guidelines Committee invited the lead author (G. N.) to form and chair a working group responsible for the module of temperature management in patients with acute ischemic stroke. The working group consisted of G. N., T. D., P. M., V. P., J. P., D. S., B. T., and T. S.; the conflicts of interest of its members are presented in Appendix S1. The working group consisted of internists, neurologists, and an information specialist; members of nursing or other disciplines were not involved in writing of these recommendations. Briefly, the steps undertaken by the working group are summarized below:

1. Formulation of the PICO questions (the acronym PICO stands for population, intervention, comparator, outcome), suggested and concluded by consensus among the members of the working group. 
2. Rating of the importance of the outcomes selected using a 9-degree scale (7-9 - critical; 4-6 - important; 1-3 - of limited importance), concluded by consensus among the members of the working group

3. Identification of all available related literature. Only randomized controlled trials (RCTs) and systematic reviews/meta-analyses were considered for the current work. Systematic literature searches of eight major bibliographic databases were performed to identify relevant studies relating to the identified PICO questions. The Cochrane Stroke Group Information Specialist (B. T.) developed the search strategies for each database using a combination of controlled vocabulary and free text terms to describe each PICO topic and performed the literature searches between December 2013 and February 2014 (Appendix S2). The following databases were searched for each PICO question: the Cochrane Stroke Group Trials Register (7), The Cochrane Library (the Cochrane Database of Systematic Reviews), the Cochrane Central Register of Controlled Trials, the Database of Reviews of Effects, and the Health Technology Database, MEDLINE (Ovid) (from 1946), EMBASE (from 1980), and Cumulative Index of Nursing and Allied Health Literature (CINAHL) (Ebsco) (from 1982).

4. Selection of eligible studies. For each PICO question, two authors independently screened the titles and abstracts of the publications identified by the corresponding electronic search and assessed the full text of potentially relevant studies.

5. Extraction of data from study reports was performed independently by two authors for each PICO question. In case that data were not reported in an eligible study, its corresponding author was contacted with the request to provide the necessary data. In case of no response, the co-authors of the study were also contacted. In case of no response by the co-authors, several reminders were sent to all authors. If again no answer was received, data were considered as missing and were not included in the analysis.

6. Analysis of extracted data using the Review Manager 5. Analysis was performed on a random-effects basis, and results are summarized as risk ratios (RR) and 95\% confidence intervals (CIs). Heterogeneity across studies was evaluated with the $I^{2}$ test. Publication bias was assessed with the help of the funnel plots.

7. Import of analyzed data into the GRADEPro software (8).

8. Grading of the quality of available evidence for each outcome, concluded by consensus among the members of the working group using the following criteria: the type of studies included, limitations in study design and methodology (i.e. risk of bias), inconsistency (or else: heterogeneity) of results, indirectness of evidence, imprecision, reporting bias, the magnitude of the treatment effect, evidence of a dose-response relation, and the effect of all plausible confounding. Quality of evidence was graded in four grades as high, moderate, low, and very low (Box 1) $(1,2,9,10)$.

9. Grading of the quality of evidence across several outcomes. When several outcomes were assessed for a clinical question, the grade for the overall quality of evidence was based on the grade for the most critical. In general, critical outcomes determine the grading of quality of evidence across different outcomes $(1,2,9,10)$.

\begin{tabular}{lll}
\hline Box 1 Grades of quality of evidence & & Symbol \\
\hline Grade & Definition & $\begin{array}{l}\text { Further research is very unlikely to change our } \\
\text { confidence in the estimate of effect. }\end{array}$ \\
\hline Moderate & $\begin{array}{l}\text { Further research is likely to have an important } \\
\text { impact on our confidence in the estimate of } \\
\text { effect and may change the estimate. }\end{array}$ & \\
& $\begin{array}{l}\text { Further research is very likely to have an } \\
\text { important impact on our confidence in the } \\
\text { estimate of effect and is likely to change the } \\
\text { estimate. }\end{array}$ & \\
Very low & $\begin{array}{l}\text { We are very uncertain about the estimate. } \\
\text { Lew }\end{array}$ & $\oplus \oplus$ \\
\hline
\end{tabular}

Box 2 Definitions and symbols of categories of strength of recommendation

\begin{tabular}{cll}
\hline $\begin{array}{l}\text { Strength of } \\
\text { recommendation }\end{array}$ & Criteria & Symbol \\
\hline $\begin{array}{c}\text { Strong for an } \\
\text { intervention }\end{array}$ & $\begin{array}{c}\text { The desirable effects of an intervention } \\
\text { clearly outweigh its undesirable } \\
\text { effects. }\end{array}$ & $\uparrow \uparrow$ \\
$\begin{array}{c}\text { Weak for an } \\
\text { intervention }\end{array}$ & $\begin{array}{c}\text { The desirable effects of an intervention } \\
\text { probably outweigh the undesirable } \\
\text { effects. }\end{array}$ & \\
$\begin{array}{c}\text { Weak against an } \\
\text { intervention }\end{array}$ & $\begin{array}{c}\text { The undesirable effects of an } \\
\text { intervention probably outweigh the } \\
\text { desirable effects }\end{array}$ & $\downarrow ?$ \\
$\begin{array}{c}\text { Strong against an } \\
\text { intervention }\end{array}$ & $\begin{array}{c}\text { The undesirable effects of an } \\
\text { intervention clearly outweigh its } \\
\text { desirable effects. }\end{array}$ & \\
\hline
\end{tabular}

10. Determination of the direction and the strength of the recommendation, concluded by consensus among the members of the working group. For each PICO question, according to the GRADE methodology, the direction of recommendation was either 'for' or 'against', and the strength of recommendation was defined as either strong or weak, taking into consideration the balance between desirable and undesirable effects and the quality of the evidence (Box 2) $(1,2,9,10)$.

11. Wording of recommendations was concluded by consensus among the members of the working group. For strong recommendations, we adapted the terminology 'we recommend...', whereas for weak recommendations, we adapted the term 'we suggest....

For each PICO question, we add an 'additional information' box just after each recommendation box to provide further information which may be of interest to the reader (11).

This has been discussed during a plenary session during the ESO-Karolinska Stroke Update Conference. This document was approved by consensus by the members of the working group for the preparation of the ESO Guidelines about temperature management in acute ischemic stroke (Appendix S1a); it was reviewed by two external reviewers (Appendix S1d), who do not carry any responsibility for its integrity. It was submitted to and approved for publication by the ESO Guidelines Committee (Appendix S1b) and the ESO Executive Committee (Appendix S1c). 


\section{Results}

The working group formulated three PICO questions, each one examining two outcomes: functional outcome and mortality. For all PICO questions, both outcomes were rated as of critical importance (9/9).

PICO1: In hyperthermic patient with acute ischemic stroke, does treatment of hyperthermia compared with no treatment of hyperthermia improve functional outcome and/or survival?

The literature search was based on the strategy shown in Appendix S2a; it was performed on 11 December 2013 and yielded 3105 items. Two authors (G. N. and V. P.) screened the titles and abstracts of these publications, retrieved 154 publications in full text, and identified two small RCTs of a total of 42 patients (Appendix S3a) $(12,13)$. The intervention tested was paracetamol in both trials $(12,13)$. For the present analysis, hyperthermia was arbitrarily defined as $\geq 38^{\circ} \mathrm{C}$.

Favorable functional outcome was assessed with the modified Rankin Scale (mRS) at one- to three-months in both trials, and was defined as a score of $\leq 3$ in the study by Hertog et al. (12) and a score of $\leq 2$ in the study by Dippel et al. (13). In the analysis of all 42 patients, there was no statistically significant difference in favorable functional outcome between patients receiving treatment for hyperthermia and controls [risk ratio (RR): 1·30, 95\% CI: $0 \cdot 78-2 \cdot 15$ ] with no sign of heterogeneity among the RCTs $\left(I^{2}: 0 \%\right.$; Fig. S1). The quality of evidence was downgraded to low due to serious imprecision of the effect estimates (Table 1). The funnel plot of the included RCTs is presented in Fig. S2.

Mortality was also assessed at one- to three-months in both trials $(12,13)$. There was no statistically significant difference in mortality between patients receiving treatment for hyperthermia and controls (RR: $0 \cdot 88,95 \%$ CI: $0 \cdot 21-3 \cdot 71$ ), with no sign of heterogeneity ( $I^{2}: 0 \%$; Fig. S1). The quality of evidence was downgraded to low due to serious imprecision (Table 1). The funnel plot of the included RCTs is presented in Fig. S2.

\section{Recommendation}

In patients with acute ischemic stroke and hyperthermia, we cannot make any recommendation for treating hyperthermia as a means to improve functional outcome and/or survival.

Quality of evidence: $L O W / \oplus \oplus$

Strength of recommendation: Weak

Additional information: Recently, the QASC trial showed that rigorous implementation of common stroke treatment protocols including fever management, dysphagia, and hyperglycemia management improved patient outcomes (14). Although it was not possible to identify directly which of the three components of this intervention exerted the beneficial effect which was actually the reason for excluding this cluster-randomized RCT from our meta-analysis (15), a multiple logistic regression analysis suggested that the main determinants were hyperglycemia and fever management (16).

Administration of antipyretics to reduce temperature in a hyperthermic patient and clinical examination and investigation to identify the cause of fever are standard of care worldwide in routine clinical practice. In this context, treatment of hyperthermia in a hyperthermic stroke patient is a rational choice to reduce temperature and relieve the symptom of discomfort associated with hyperthermia; however, as analyzed above, existing data are very limited to show any effect (either beneficial or detrimental) in stroke patients on hard clinical outcomes like functional outcome or mortality. Further randomized trials are needed.

PICO2: In normothermic patients with acute ischemic stroke, does prevention of hyperthermia with antipyretics compared with no prevention of hyperthermia improve functional outcome and/or survival?

The literature search was based on the strategy shown in Appendix S2b; it was performed on 8 January 2014 and yielded 814 items. Two authors (T. D. and P. M.) screened the titles and abstracts of these publications and retrieved 13 publications in full, of which four were RCTs of a total of 1354 patients (Appendix S3b) $(12,13,17,18)$. The antipyretics tested were paracetamol and/or ibuprofen. Normothermia was defined as $<38^{\circ} \mathrm{C}$ in three studies $(12,13,18)$ and $<38 \cdot 5^{\circ} \mathrm{C}$ in one study $(17)$.

Favorable functional outcome was assessed in three trials and defined as an mRS score at one- to three-months of $\leq 3$ in the study by Hertog et al. (12) and $\leq 2$ in the two studies by Dippel et al. $(13,18)$. In the analysis of 1323 patients, there was no statistically significant difference in favorable functional outcome between patients receiving preventive measures for hyperthermia and controls (RR: 1.02, 95\% CI: 0.94-1·10) with no sign of heterogeneity $\left(I^{2}: 0 \%\right)$ (Fig. S3). The quality of evidence was graded as high (Table 2). The funnel plot of the included RCTs is presented in Fig. S4.

Four RCTs assessed mortality at one- to three-months after stroke $(12,13,17,18)$. The analysis of 1354 patients showed no statistically significant difference in mortality between patients receiving preventive measures for hyperthermia and controls (RR: $0 \cdot 96,95 \%$ CI: $0 \cdot 74-1 \cdot 23)$ with no sign of heterogeneity $\left(I^{2}\right.$ : $0 \%$ ) (Fig. S3). The quality of evidence was downgraded to moderate due to serious imprecision (Table 2). The funnel plot of the included RCTs is presented in Fig. S4.

\section{Recommendation}

In patients with acute ischemic stroke and normothermia, we do not recommend routine prevention of hyperthermia with antipyretics as a means to improve functional outcome and/or survival.

Quality of evidence: Moderate $/ \oplus \oplus \oplus$

Strength of recommendation: Weak/ $\downarrow$ ?

Additional information: This recommendation refers only to targeted temperature management approaches like administration of antipyretics and not to routine best-care practices like assessment of dysphagia and prevention of aspiration pneumonia, prevention of urinary tract infections, and other infections and prevention of pressure ulcers, all of which may induce hyperthermia. Also, a benefit cannot be excluded in specific patient populations (e.g. those with a body temperature in the first 12 hours of stroke onset of $37 \cdot 0^{\circ} \mathrm{C}$ or higher (19)); therefore, new clinical trials are recommended. 



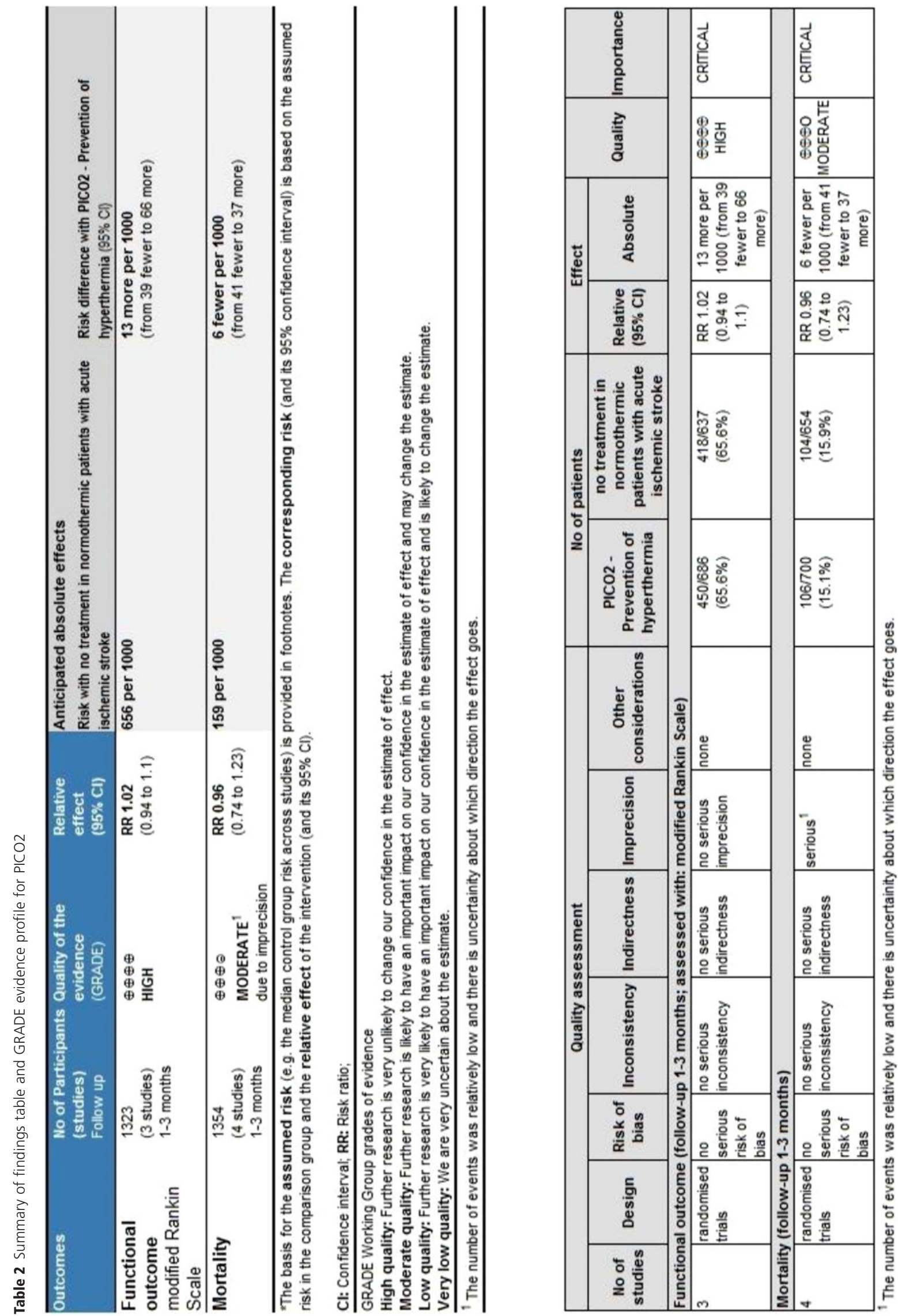
PICO3: In patients with acute ischemic stroke, does induction of hypothermia compared with no induction of hypothermia improve functional outcome and/or survival?

The literature search was based on the strategy shown in Appendix S2c; it was performed on 11 February 2014 and yielded 4790 items. Two authors (J. P. and D. S.) screened the titles and abstracts of these publications and retrieved 55 publications in full, of which six RCTs were identified, including a total of 252 patients (Appendix S3c) (20-24).

Favorable functional outcome was assessed with the mRS at one- to three-months in five studies, and defined as $\leq 1$ in the studies by Bi et al. (21) and Hemmen et al. (23) and $\leq 2$ in the studies by Piironen et al. (20), Ovesen et al. (24) and De Georgia et al. (22). The analysis included 227 patients and showed no statistically significant difference in favorable functional outcome between patients treated with induction of hypothermia and controls (RR: 0.92, 95\% CI: 0.63-1.33), with no sign of heterogeneity $\left(I^{2}: 0 \%\right)$ (Fig. S5). The quality of evidence was graded as low because of serious risk of bias and serious imprecision (Table 3 ). The funnel plot of the included RCTs is presented in Fig. S6.

Mortality was assessed at one- to three-months in all six trials (20-25). The analysis included 252 patients, and there was no statistically significant difference in mortality between patients receiving hypothermia and controls (RR: 1·20, 95\% CI: 0.65$2 \cdot 22)$, with no sign of heterogeneity $\left(I^{2}: 0 \%\right)$ (Fig. S5). The quality of evidence was graded as very low because of the serious risk of bias and very serious imprecision (Table 3). The funnel plot of the included RCTs is presented in Fig. S6.

\section{Recommendation}

In patients with acute ischemic stroke, we do not recommend induction of hypothermia as a means to improve functional outcome and/or survival.

Quality of evidence: Very low $/ \oplus$

Strength of recommendation: Weak/ $\downarrow$ ?

Additional information: Currently available data are limited to guide a strong clinical recommendation, but the ongoing EuroHYP-1 (26) and ICTuS 2/3 (27) are expected to provide further insight. We strongly encourage recruitment of eligible patients to these RCTs.

\begin{tabular}{ll}
\hline Recommendation & $\begin{array}{l}\text { Quality of } \\
\text { evidence/strength } \\
\text { of recommendation }\end{array}$ \\
\hline $\begin{array}{l}\text { 1. In patients with acute ischemic stroke and } \\
\text { hyperthermia, we cannot make any }\end{array}$ & $\oplus \oplus /$ ? \\
recommendation for treating hyperthermia as a \\
means to improve functional outcome and/or \\
survival. \\
2. In patients with acute ischemic stroke and \\
normothermia, we do not recommend routine \\
prevention of hyperthermia with antipyretics as \\
a means to improve functional outcome and/or \\
survival. \\
3. In patients with acute ischemic stroke, we do \\
not recommend induction of hypothermia as a \\
means to improve functional outcome and/or \\
survival.
\end{tabular}

\section{Discussion}

The currently available data about temperature management in acute ischemic stroke are limited, and the strengths of the recommendations are therefore weak. Based on the existing data, we cannot make any recommendation for treating hyperthermia as a means to improve functional outcome and/or survival in patients with acute ischemic stroke and hyperthermia; we do not recommend routine prevention of hyperthermia with antipyretics as a means to improve functional outcome and/or survival in patients with acute ischemic stroke and normothermia, and we do not recommend routine induction of hypothermia as a means to improve functional outcome and/or survival in patients with acute ischemic stroke. The limitations of the available data call for recruitment of patients into ongoing and future RCTs.

Hyperthermia is a frequent complication in up to $50 \%$ of patients with acute ischemic stroke $(3,4)$ and has been shown to be associated with poor outcome $(5,6)$. This effect may be mediated by increased production of excitotoxins and oxygen radicals, destabilization of cellular membranes, and abnormal electrical depolarizations (28-32). Hyperthermia also increases oxygen demand and may aggravate cardiac or pulmonary insufficiency, and it may also induce mental changes in patients with brain disease. It is a common practice worldwide - not only in patients with acute ischemic stroke - to treat hyperthermia with antipyretics, mainly with paracetamol (4). The recent AHA/ASA guidelines for the early management of patients with acute ischemic stroke recommend that antipyretic medication should be administered to lower temperature in patients with stroke and hyperthermia. This recommendation is graded as class I/level C and is based on consensus opinion of experts and case studies or standard of care (33). We cannot make any recommendation for treating patients with acute ischemic stroke and hyperthermia as a means of improving functional outcome and/or survival, but this does not preclude giving antipyretics to relieve the symptom of discomfort associated with hyperthermia. Since there is absence of evidence, we could, according to GRADE terminology, also have opted for a recommendation against treating hyperthermia in patients with acute ischemic stroke. However, since there is some evidence on possible benefits and very little evidence against treatment, we chose the more neutral term above. The recommendations of the ESO and the AHA/ASA may seem contradictory; however, it needs to be pointed out that the outcomes are different between the two recommendations (functional outcome and/or survival in the ESO guidelines, reduction of temperature in the AHA/ASA guidelines). Obviously, the administration of antipyretic medication in a hyperthermic patient with acute ischemic stroke seems a rational choice given that it reduces the patient's temperature and relieves the feeling of discomfort. The strength of the ESO recommendation is weak, which implies that little evidence is needed to change the recommendation. In the absence of a clear recommendation for critical outcomes, a strong recommendation for less critical outcomes (such as patient comfort) may change the overall recommendation. However, for this review, we have chosen only to consider critical outcomes like functional outcome and survival, and in the 'additional information box', we have 


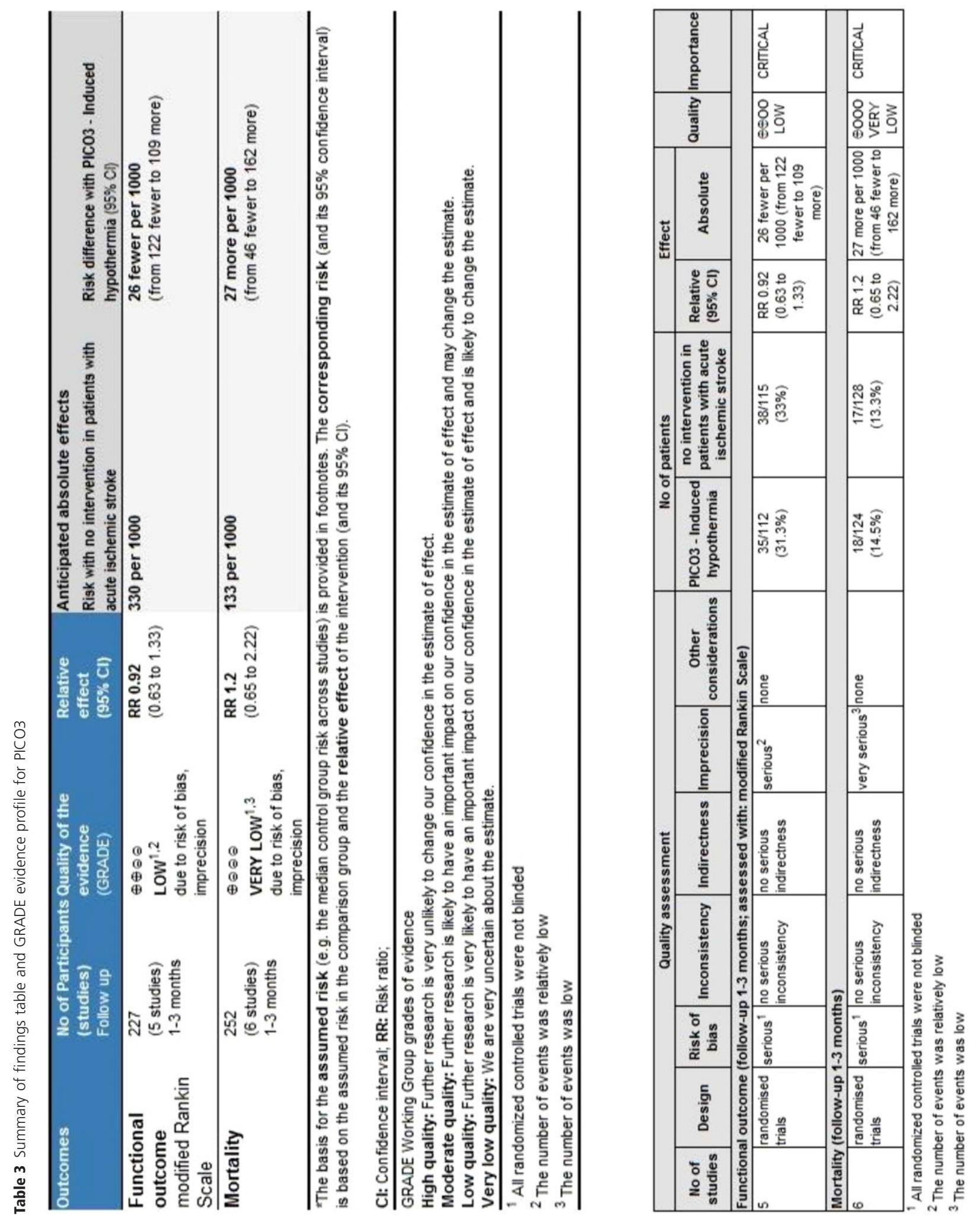


explained that administration of an antipyretic medication can still be a reasonable approach. For the next version of this recommendation, the working group may decide to include other less important outcomes.

The same precautions must be taken when considering our suggestion against prevention of hyperthermia in normothermic patients as this refers only to routine prevention with antipyretics and not to best care practices like assessment of dysphagia and prevention of aspiration pneumonia, prevention of urinary tract infections, and prevention of pressure ulcers, all of which may induce hyperthermia. There is also indirect evidence that induction of hypothermia is effective in patients with hypoxic encephalopathy after cardiac arrest (34,35), after perinatal asphyxia (36), and after focal cerebral ischemia in animal models $(37,38)$, but we found no direct evidence that induction of hypothermia is effective in patients with acute ischemic stroke; well-designed and adequately powered RCTs like the ongoing EuroHYP-1 (26) and ICTuS 2/3 (27) are needed to provide further insight.

The main strengths of this work are the systematic review across several databases to identify potential eligible studies and the implementation of the GRADE system, which is adopted by many healthcare organization worldwide because of its advantages like the clear separation between quality of evidence and strength of recommendations, the explicit evaluation of the importance of outcomes, the explicit criteria for downgrading and upgrading the ratings of the quality of evidence, and the transparent process of moving from evidence to recommendations $(1,2,9,10)$. Finally, we added an 'additional information' box after each recommendation box to provide further information which may be of value for the reader. We chose to include only RCTs in order to avoid the inherent limitations of observational studies like selection bias and unmeasured confounding. Also, we chose to study only hard outcomes like functional outcome and mortality. Given the limited evidence available, for the next version of this document, the working group may decide to include also observational studies, patients' preferences and values, as well as other less important outcomes. Finally, for the next version of this document, the working group may expand to include members from other disciplines like nursing.

In conclusion, the currently available data about temperature management in acute ischemic stroke are limited, and therefore, the strength of recommendations is weak. We call for new RCTs as well as recruitment of eligible patients in ongoing RCTs to allow for better-informed recommendations in the future.

\section{References}

1 Guyatt GH, Oxman AD, Kunz R et al. Going from evidence to recommendations. BMJ 2008; 336:1049-51.

2 Guyatt GH, Oxman AD, Vist GE et al. Grade: an emerging consensus on rating quality of evidence and strength of recommendations. BMJ 2008; 336:924-6.

3 Castillo J, Davalos A, Marrugat J, Noya M. Timing for fever-related brain damage in acute ischemic stroke. Stroke 1998; 29:2455-60.

4 Drury P, Levi C, McInnes E et al. Management of fever, hyperglycemia, and swallowing dysfunction following hospital admission for acute stroke in New South Wales, Australia. Int J Stroke 2014; 9:23-31.
5 Prasad K, Krishnan PR. Fever is associated with doubling of odds of short-term mortality in ischemic stroke: an updated meta-analysis. Acta Neurol Scand 2010; 122:404-8.

6 Azzimondi G, Bassein L, Nonino F et al. Fever in acute stroke worsens prognosis. A prospective study. Stroke 1995; 26:2040-3.

7 The Editorial Team. Cochrane stroke group. About the Cochrane collaboration (Cochrane review groups (CRGs)) 2013, issue 11. Art. No.: Stroke.

8 Gradepro. [computer program]. Version 3.2 for windows. Jan Brozek, Andrew Oxman, Holger Schünemann, 2008.

9 Guyatt GH, Oxman AD, Kunz R et al. Incorporating considerations of resources use into grading recommendations. BMJ 2008; 336:1170-3.

10 Guyatt GH, Oxman AD, Kunz R et al. What is 'quality of evidence' and why is it important to clinicians? BMJ 2008; 336:995-8.

11 Pocock SJ, Ware JH. Translating statistical findings into plain English. Lancet 2009; 373:1926-8.

12 Hertog HM, Worp HB, Gemert HM et al. The paracetamol (acetaminophen) in stroke (pais) trial: a multicentre, randomised, placebocontrolled, phase iii trial. Lancet Neurol 2009; 8:434-40.

13 Dippel DW, van Breda EJ, van Gemert HM et al. Effect of paracetamol (acetaminophen) on body temperature in acute ischemic stroke: a double-blind, randomized phase ii clinical trial. Stroke 2001; 32:160712.

14 Middleton S, McElduff P, Ward J et al. Implementation of evidencebased treatment protocols to manage fever, hyperglycaemia, and swallowing dysfunction in acute stroke (QASC): A cluster randomised controlled trial. Lancet 2011; 378:1699-706.

15 Drury P, Levi C, D'Este C et al. Quality in acute stroke care (QASC): process evaluation of an intervention to improve the management of fever, hyperglycemia, and swallowing dysfunction following acute stroke. Int J Stroke 2013; 9:766-76.

16 Middleton S, Drury P, Levi CR et al. What processes of clinical care are associated with 90-day survival and independence? Results from the quality in acute stroke care QASC trial. Int J Stroke 2012; 7:25.

17 Kasner SE, Wein T, Piriyawat P et al. Acetaminophen for altering body temperature in acute stroke. A randomized clinical trial. Stroke 2002; 33:130-5.

18 Dippel DW, Breda EJ, Worp HB et al. Effect of paracetamol (acetaminophen) and ibuprofen on body temperature in acute ischemic stroke pisa, a phase ii double-blind, randomized, placebo-controlled trial [isrctn98608690]. BMC Cardiovasc Disord 2003; 3:2.

19 de Ridder IR, de Jong FJ, den Hertog HM et al. Paracetamol (acetaminophen) in stroke 2 (pais 2): protocol for a randomized, placebocontrolled, double-blind clinical trial to assess the effect of high-dose paracetamol on functional outcome in patients with acute stroke and a body temperature of 36.5 degrees c or above. Int J Stroke 2015; 10:457-62.

20 Piironen K, Tiainen M, Mustanoja S et al. Mild hypothermia after intravenous thrombolysis in patients with acute stroke: a randomized controlled trial. Stroke 2014; 45:486-91.

21 Bi M, Ma Q, Zhang S et al. Local mild hypothermia with thrombolysis for acute ischemic stroke within a 6-h window. Clin Neurol Neurosurg 2011; 113:768-73.

22 De Georgia MA, Krieger DW, bou-Chebl A, Devlin TG, Jauss M, Davis SM. Cooling for acute ischemic brain damage (cool aid). A feasibility trial of endovascular cooling. Neurology 2004; 63:312-7.

23 Hemmen TM, Guluma KZ, Wijman CA, Cruz-Flores S, Meyer BC, Rapp KS. Intravenous thrombolysis plus hypothermia for acute treatment of ischemic stroke (ICTuS-L). Stroke 2006; 37:706.

24 Ovesen C, Brizzi M, Pott FC et al. Feasibility of endovascular and surface cooling strategies in acute stroke. Acta Neurol Scand 2013; 127:399-405.

25 Els T, Oehm E, Voigt S, Klisch J, Hetzel A, Kassubek J. Safety and therapeutical benefit of hemicraniectomy combined with mild hypothermia in comparison with hemicraniectomy alone in patients with malignant ischemic stroke. Cerebrovascr Dis. 2006; 21:79-85. 
26 van der Worp HB, Macleod MR, Bath PM et al. Eurohyp-1: European multicenter, randomized, phase III clinical trial of therapeutic hypothermia plus best medical treatment vs. Best medical treatment alone for acute ischemic stroke. Int J Stroke 2014; 9:642-5.

27 Lyden PD, Hemmen TM, Grotta J, Rapp K, Raman R. Endovascular therapeutic hypothermia for acute ischemic stroke: Ictus $2 / 3$ protocol. Int J Stroke 2014; 9:117-25.

28 Ginsberg MD, Busto R. Combating hyperthermia in acute stroke: a significant clinical concern. Stroke 1998; 29:529-34.

29 Busto R, Globus MY, Dietrich WD, Martinez E, Valdes I, Ginsberg MD. Effect of mild hypothermia on ischemia-induced release of neurotransmitters and free fatty acids in rat brain. Stroke 1989; 20:904-10.

30 Dietrich WD, Alonso O, Halley M, Busto R. Delayed posttraumatic brain hyperthermia worsens outcome after fluid percussion brain injury: a light and electron microscopic study in rats. Neurosurgery 1996; 38:533-41.

31 Minamisawa H, Smith ML, Siesjo BK. The effect of mild hyperthermia and hypothermia on brain damage following 5, 10, and 15 minutes of forebrain ischemia. Ann Neurol 1990; 28:26-33.

32 Wass CT, Lanier WL, Hofer RE, Scheithauer BW, Andrews AG. Temperature changes of $>$ or $=1$ degree $\mathrm{c}$ alter functional neurologic outcome and histopathology in a canine model of complete cerebral ischemia. Anesthesiology 1995; 83:325-35.

33 Jauch EC, Saver JL, Adams HP Jr et al. Guidelines for the early management of patients with acute ischemic stroke: a guideline for healthcare professionals from the American Heart Association/American Stroke Association. Stroke 2013; 44:870-947.

34 Hypothermia after Cardiac Arrest Study G. Mild therapeutic hypothermia to improve the neurologic outcome after cardiac arrest. $N$ Engl J Med 2002; 346:549-56.

35 Bernard SA, Gray TW, Buist MD et al. Treatment of comatose survivors of out-of-hospital cardiac arrest with induced hypothermia. $N$ Engl J Med 2002; 346:557-63.

36 Jacobs SE, Berg M, Hunt R, Tarnow-Mordi WO, Inder TE, Davis PG. Cooling for newborns with hypoxic ischaemic encephalopathy. Cochrane Database Syst Rev 2013; (1) CD003311.

37 van der Worp HB, Sena ES, Donnan GA, Howells DW, Macleod MR. Hypothermia in animal models of acute ischaemic stroke: a systematic review and meta-analysis. Brain 2007; 130:3063-74.
38 van der Worp HB, Macleod MR, Kollmar R. European Stroke Research Network for H. Therapeutic hypothermia for acute ischemic stroke: ready to start large randomized trials? J Cereb Blood Flow Metab 2010; 30:1079-93.

\section{Supporting information}

Additional Supporting Information may be found in the online version of this article at the publisher's web-site:

Figure S1. Forest plots for PICO1.

Figure S2. Funnel plots for PICO1.

Figure S3. Forest plots for PICO2.

Figure S4. Funnel plots for PICO2.

Figure S5. Forest plots for PICO3.

Figure S6. Funnel plots for PICO3.

Appendix S1. Conflicts of interest of the members of the working group (a), ESO Guideline Committee (b), ESO executive committee (c) and external reviewers (d).

Appendix S2. Search strategies for PICO1 (a), PICO 2 (b), and PICO3 (c).

Appendix S3. Flow diagrams for PICO1 (a), PICO2 (b), and $\mathrm{PICO} 3$ (c). 